\title{
El CRITERIO DE EMPIRICAL GROUNDING EN EL ESTRUCTURALISMO EMPIRISTA
}

\author{
SUSANA LUCERO \\ BRUNO BORGE
}

\begin{abstract}
Van Fraassen's Empiricist Structuralism has been threatened by what is known as the loss of reality objection: the pre-eminence of pragmatic factors in the scientific representation of phenomena seems to neglect the role of the world itself in the adequacy of theories. One of van Fraassen's weapons for addressing to this objection is a set of methodological rules he calls criterion of empirical grounding. In the present work we question the effectiveness of this criterion (and the methodological strategy in general) to avoid objections such as the loss of reality.
\end{abstract}

Keywords: Empiricist structuralism; empirical grounding; scientific representation; scientific models.

\section{Introducción}

Dentro del giro estructuralista protagonizado por la filosofía de la ciencia del siglo XX se destaca la propuesta del Estructuralismo Empirista de van Fraassen (en adelante EE), una concepción preanunciada en su clásico libro The Scientific Image (1980) y desarrollada de manera minuciosa y fructífera en su última célebre obra Scientific Representation: Paradoxes of Perspective (2008). Cuestiones relativas a la representación, las estructuras y la experiencia adquieren en esta obra un papel preponderante. En particular, asistimos a una elaborada y muy rica teoría de la representación que incorpora los factores pragmáticos como elementos imprescindibles y determinantes en la actividad de representar. Uno de los problemas más acuciantes abordados por su autor es el desafío de explicar, de manera plausible y convincente, la relación entre las teorías y el mundo. En otras palabras: cómo es posible que un modelo teórico, que es una estructura matemática, puede representar un fenómeno físico, que no es una entidad matemática, en términos de una relación de isomorfismo estructural. Para resolver la brecha entre la teoría - entendida como familia de modelos - y los fenómenos del mundo, van Fraassen interpone otra estructura mediadora, el modelo de datos. En realidad, el modelo de datos es resultado de mediciones singulares tomadas cada una en un momento particular. Estas mediciones son procesadas estadísticamente y sistematizadas, el producto final de este proceso es un modelo de superficie. El modelo de superficie "idealiza", de hecho, el resumen de mediciones tomadas, de modo tal que reemplaza el total de frecuencias relativas por mediciones

Principia 21(3): 473-482 (2017).

Published by NEL — Epistemology and Logic Research Group, Federal University of Santa Catarina (UFSC), Brazil. 
que tienen un rango continuo de valores (van Fraassen 2008, p.167). En este trabajo - y siguiendo una sugerencia de van Fraassen - usaremos el término "modelo de datos" para referirnos al resultado final del proceso de medición. El modelo de datos cumple la función de conectar los dos términos más importantes de la trilogía: el modelo teórico y los fenómenos. La relación de isomorfismo estructural que se establece entre el modelo teórico y el modelo de datos resulta entonces admisible porque convergen dos estructuras matemáticas.

A este respecto van Fraassen introduce una distinción relevante: separa los fenómenos de las apariencias. Los fenómenos son los objetos, eventos y procesos directamente observables, mientras que las apariencias son construidas por nuestra actividad intelectual independiente (van Fraassen 2008, p.269); luego, los modelos de datos integran las apariencias, es decir no constituyen, estrictamente hablando, los fenómenos indagados por la teoría sino que son más bien sus representantes matemáticos. Dos cuestiones importantes surgen en este planteo: en primer lugar ¿cuál es la relación particular entre las apariencias (el modelo de datos) y el fenómeno? Y en segundo término ¿en qué sentido puede afirmarse que el modelo teórico representa el fenómeno y no el modelo de datos construido por el usuario? Nótese que la relación de representación no es transitiva: si $A$ representa $B$ y $B$ representa $C$, de aquí no se sigue que $A$ representa $C$. Consciente de estas dificultades, van Fraassen adelanta una objeción que podría formular un crítico de filiación realista: en verdad el modelo teórico no representa el fenómeno en estudio, y nosotros queremos que lo represente. Van Fraassen denomina este escollo la objeción de pérdida de la realidad. (van Fraassen 2008, p.258).

Mediante una argumentación centrada esencialmente en la pragmática de la representación, (lo que denomina la movida wittgensteiniana), el filósofo afirma haber resuelto - y aun disuelto - el problema de pérdida de la realidad. Con este propósito recurre a la indexicalidad del lenguaje, un fenómeno que aparece también en el acto de representar. Así, el modelo de datos construido por los usuarios, por una parte, y el fenómeno físico a partir del cual se elaboró el modelo, por otra, son lo mismo para nosotros (los usuarios). Se ha establecido, de este modo, una equivalencia pragmática entre los dos términos de la relación. Si a un usuario se le ocurriera negar que la teoría representa el fenómeno en estas circunstancias, incurriría en una contradicción pragmática de la forma: "el modelo de datos representa el fenómeno pero, hasta donde yo sé, no lo representa” (van Fraassen 2008, p.257). La teoría ha logrado anclar, después de todo, en el mundo real.

El argumento de la indexicalidad ha sido objeto de agudas críticas por parte de varios autores. En otro lugar hemos formulado nuestras propias objeciones y demostrado que - a diferencia de lo que afirmó van Fraassen - el problema de la relación entre la teoría y el mundo no queda resuelto con el recurso al argumento de la indexicalidad (Borge y Lucero (2018)). En este trabajo vamos a referirnos a otro aspecto

Principia 21(2): 473-482 (2017). 
importante de la propuesta ofrecida en el marco del EE: cómo garantizar una relación apropiada y plausible entre la construcción de modelos y el objetivo general de adecuación empírica asignado a las teorías. En el año 2014 van Fraassen publica el artículo "The Criterion of Empirical Grounding in the Sciences" con vistas a esclarecer esta cuestión. Esta vez el enfoque que adopta para fundamentar su perspectiva es de carácter metodológico. Nuestro propósito es mostrar que el recurso a la metodología constituye una condición necesaria pero no alcanza por sí misma para garantizar el vínculo de la teoría con la meta asignada a la ciencia, la adecuación empírica; por ser éste un objetivo de carácter primordialmente epistémico.

\section{Acerca del criterio vanfraasseano de Empirical Grounding}

Más allá de las dificultades asociadas a la objeción de la pérdida de la realidad, sostenemos que dos problemas adicionales socavan el planteo del EE. El primer problema deriva de la afirmación conjunta de una serie de compromisos que, por diversas razones, van Fraassen incorpora en el EE. Llamaremos a esta primera cuestión el problema del conocimiento objetivo. La segunda cuestión constituye lo que denominamos el problema de las restricciones de la representación.

Previo a comenzar nuestra argumentación es preciso entonces repasar los principales compromisos que van Fraassen pretende incorporar a su EE.

Primero, el EE, al igual que el Empirismo Constructivo, se enmarca en un Realismo del Sentido Común que ha sido ajeno a la tradición empirista. En tal sentido, van Fraassen acepta que podemos conocer de modo no problemático entidades del sentido común. Segundo, se rechaza de plano el Realismo Estructural (tanto en su versión epistémica como óntica) y toda metafísica que afirme la existencia de estructuras naturales, dadas o privilegiadas en lo fenómenos. Tercero, y a pesar de lo anterior, el EE se compromete con una concepción estructural del conocimiento científico. Si bien rechaza toda forma de realismo acerca de estructuras, el conocimiento científico se limita a descripciones estructurales de los fenómenos (y, claro está, solo de los fenómenos). Cuarto, esta doctrina está acompañada de una visión pragmática de la representación: nada es una representación sino en el sentido en que es usada por alguien a los efectos de representar alguna cosa de tal o cual manera. Quinto, el EE pretende salvar, incluso en este contexto, una distinción entre representaciones correctas e incorrectas. En otras palabras, no cualquier modelo de datos es adecuado a cualquier fenómeno.

Principia 21(2): 473-482 (2017). 


\subsection{Representación, conocimiento objetivo y el problema de las restricciones metodológicas}

Veamos primeramente el problema del conocimiento objetivo. El rechazo del realismo estructural, tanto en la versión epistémica de Worrall como en la versión óntica de Ladyman y French, lleva consigo el rechazo de la existencia de estructuras relacionales objetivas o naturales en el mundo. Para van Fraassen, los fenómenos que la teoría intenta salvar carecen de una estructura intrínseca. Más bien son los científicos quienes atribuyen estructura a los fenómenos reales al construir las apariencias (los modelos de datos). Sostiene además que no hay nada en una estructura abstracta que la determine como el modelo adecuado para representar cierto fenómeno y, por otra parte, el fenómeno en sí mismo carece de una estructura objetiva que pueda ser seleccionada como la interpretación privilegiada. La consecuencia de estas postulaciones es que todo el peso de la adecuación entre el modelo de datos y los fenómenos recae sobre el modo en que el científico construye las apariencias, es decir, sobre su decisión de representar el fenómeno de cierta manera y de acuerdo con ciertos intereses. Esta estrategia parece poner en peligro la objetividad del conocimiento, éste es el primer problema que se deriva de la afirmación conjunta de los compromisos asumidos.

El segundo problema que surge en conexión con el anterior se refiere a las restricciones que deben imponerse a la representación a fin de contar con un criterio apropiado para distinguir entre representaciones adecuadas y no adecuadas. Puesto que no hay diferencia en la práctica entre atribuir una estructura a los fenómenos y conocer la estructura de los fenómenos, ¿cuál podría ser la base para sustentar una distinción entre representaciones adecuadas e inadecuadas? Van Fraassen ofrece una solución a este problema: propone una fundamentación de carácter metodológico para sustentar la distinción y lo hace enunciando un criterio puramente empirista. Inspirado en la obra de Weyl (1927[1693]), denomina su propuesta Criterion of Empirical Grounding (CEG).

Buena parte de la motivación para este criterio está dada por el reconocimiento de la importancia de los procesos de medición en la construcción de los modelos y de su relación problemática con la teoría que obra de marco para dichos procesos. "[S]i un proceso es o no una medición, y si lo es, qué es lo que mide, son preguntas que tienen, en general, respuesta solo en relación con una teoría". Este enfoque, que partiendo de la teoría especifica la clase de mediciones que se van a realizar, se constituye como un enfoque "desde arriba". ${ }^{1}$ Sin embargo, el carácter teóricamente dependiente de esos procesos no debe arrojarnos a "temores escépticos", dado que dichas "respuestas, proporcionadas por la teoría, son parte de lo que permite a una teoría cumplir con el requisito estricto de fundamentación empírica [empirical grounding]". (van Fraassen 2014, p.81). El CEG impone, de este modo, profundas 
restricciones al desarrollo de modelos de datos mediante la aplicación de reglas metodológicas precisas, tanto respecto de su relación con la teoría como de su anclaje en el sistema real. No cualquier modelo es adecuado para cualquier fenómeno. El problema del conocimiento objetivo y el problema de las restricciones a la representación quedarían así neutralizados.

Hay algunas razones para dudar de la contundencia de este resultado. El CEG puede limitar severamente el rango de modelos de datos adecuados para un fenómeno y asegurar que la "contaminación teórica" de la medición no afecte su referencia objetiva, pero así y todo continúa siendo un problema cómo caracterizar una relación de representación epistémicamente relevante entre una entidad capaz de brindar un conocimiento puramente estructural (el modelo de datos), y algo que carece de toda estructura (el fenómeno). Ignoraremos aquí estas dificultades y supondremos que el CEG constituye una herramienta que es en principio capaz de lidiar con el problema del conocimiento objetivo. Nos abocaremos, en cambio, a responder dos preguntas concernientes a su lugar, función y eficacia en el marco del EE respecto del problema de las restricciones a la representación: primero, ¿qué es CEG? Segundo, ¿cuáles son las razones últimas para adoptarlo?

La primera de esas preguntas encuentra una respuesta sencilla. El CEG es una limitación metodológica, una "restricción normativa respecto de qué cuenta como una medición, o como una medición de qué" (van Fraassen 2014, p.97). El rumbo que toman los argumentos de van Fraassen puede anticiparse sin demasiado esfuerzo si se atiende a la evolución de su filosofía. El EE podría solucionar el problema de lograr un anclaje satisfactorio en la experiencia restringiendo el rango de modelos de datos adecuados para contrastar la teoría y asegurando una independencia relativa de los procesos de medición y construcción de modelos respecto de los principios teóricos que los guían.

Algunos realistas científicos han modelado una metodología que garantice una relación plausible entre la teoría y el diseño empírico. Con este fin, sus propuestas metodológicas se centran en los conceptos de confirmación/corroboración. El éxito predictivo de la teoría se interpreta como un signo de su verdad probable o de su aproximación a la verdad. La acumulación de confirmaciones experimentales y la capacidad de anticipar nuevos tipos de fenómenos son incluso leídas como señales de la confiabilidad de las teorías de bajo nivel acerca de lo observable, aquellas que determinan en parte la construcción de modelos de datos. Pero esta estrategia, tomada en su totalidad, no sería un camino posible para el defensor del EE, pues su objetivo no es alcanzar la verdad aproximada o la confirmación de la teoría sino "salvar los fenómenos". No obstante, el vínculo con la experiencia ha de mantenerse como uno de fundamentación (grounding). Van Fraassen reconoce esta situación sin reservas:

[el CEG] constituye una ruptura con la opinión de que el científico se ocupa

Principia 21(2): 473-482 (2017). 
de confirmar hipótesis teóricas, o de justificar creencias. A este respecto mi posición se asemeja a la de Frederick Suppes (1993), según la cual el científico se ocupa de credencializar en lugar de confirmar hipótesis, aunque no en su relación con el constructivismo social. (2014, p.97)

Esta situación nos plantea la siguiente pregunta: ¿qué justificación puede encontrar el defensor del EE para la adopción del CEG como estándar metodológico? Van Fraassen no va más allá de proponer su criterio en los términos ya mencionados, y no ofrece ninguna justificación para ello. No es difícil, sin embargo, imaginar dos respuestas posibles a dicha pregunta, respuestas que se enmarcan enteramente en la pragmática de la representación: (i) no hay de hecho razones para brindar una justificación más allá del hecho de que el CEG forma parte de la práctica científica, lo que sería una justificación basada en el uso; (ii) la razón para su adopción reside en su eficacia para aumentar el grado de adecuación empírica de las teorías, una justificación epistémica.

Consideremos primero (i), en una formulación más elaborada: no es necesario aventurarse en las oscuras aguas de la especulación metafísica, en la semántica o la epistemología para dar cuenta de la pertinencia del criterio. El CEG es parte de la práctica científica, impone a ésta tan solo restricciones normativas. En tanto conjunto de reglas, puede ser justificado apelando simplemente a consideraciones pragmáticas y metodológicas, derivadas de convenciones inherentes a dicha práctica.

Esta primera respuesta esconde un error fundamental. Es cierto que la formulación de un criterio normativo para la práctica científica no involucra, en principio, ninguna tesis acerca de qué cosas existen, ni cómo o con qué medios se las conoce. Es cierto también que las decisiones convencionales son comunes en la práctica científica, al punto de que muchas veces modelan dicha práctica y los criterios que la rigen. Sin embargo, la metodología es eficaz solo si es adecuada para los fines propuestos; en otras palabras, las reglas metodológicas no tienen valor intrínseco sino instrumental. De manera que si desde la etapa del Empirismo Constructivo hasta la concepción más reciente del EE se ha mantenido para la ciencia el objetivo de adecuación empírica, la metodología tiene que ser funcional a esa meta. Si bien en tanto objetivo es mucho más modesto que la verdad aproximada de las teorías, no deja de tratarse de un objetivo epistémico: la ciencia persigue "una continua acumulación de conocimiento empírico a lo largo del cambio teórico" (van Fraassen 2006, p.537). Así que, aun cuando sea correcto afirmar que el CEG no implica por sí compromisos semánticos, epistémicos o metafísicos, las razones para su adopción quedan supeditadas al objetivo epistémico. La práctica científica tendría que ser acorde con la meta propuesta, sin embargo las decisiones y reglas metodológicas, sin otro sustento que la propia práctica, se revelan insuficientes para justificar el objetivo de adecuación empírica.

Por su parte, (ii) afirma precisamente que el CEG es la metodología más indicada 
para alcanzar la creciente adecuación empírica de nuestras teorías. Aunque orientada al objetivo epistémico correcto, esta estrategia resulta también inconveniente. Las razones de su fracaso dependen de cómo se entienda el término 'adecuación empírica'. Si lo que allí significa 'adecuación empírica' es la mera relación de isomorfismo entre el modelo de datos y las subestructuras empíricas de la teoría que se está contrastando, el CEG resulta tan deseable como cualquier estrategia ad hoc que permita construir el modelo de datos apropiado para asegurar dicho isomorfismo. Si el objetivo es solo establecer una función entre dos estructuras matemáticas, cualquier estrategia que permita acomodar una relación de isomorfismo estructural entre los relata resultará igualmente válida. Pero no puede ser eso lo que se quiere expresar con 'adecuación empírica', sino más bien el hecho de que la relación de isomorfismo entre ambas estructuras matemáticas se da en una circunstancia tal en la que una de ellas, el modelo de datos, se hace "transparente" — por así decirlo — generando así un vínculo entre la teoría y la experiencia. Es decir, las subestructuras empíricas de la teoría, por medio del modelo de datos, resultan adecuadas a algún aspecto de los fenómenos mismos, de este modo se justifica una distinción entre representaciones adecuadas e inadecuadas. Las representaciones inadecuadas (misrepresentations) son aquellas en las que la isomorfía estructural pretendida resulta fallida. Pero la pregunta obligada es, ¿cómo es que CEG asegura que eso suceda?

Es imperioso recordar que los fenómenos, en sí mismos, no tienen estructura. Pero incluso si ello no fuera un problema o pudiese superarse con el argumento de la indexicalidad (o algún otro), la pregunta permanece: ¿por qué el CEG es el recurso más adecuado para garantizar la relación entre teoría y fenómeno? Ello no puede simplemente afirmarse. Hacerlo equivaldría a pretender que el CEG generase fundamentación empírica solo por llamarse de ese modo. Consideremos como ejemplo uno de los aspectos regulados por el CEG y preguntemos “¿Por qué la concordancia de valores para una misma variable - determinados sobre la base de conexiones teóricamente postuladas - por medios experimentales diferentes es un criterio más adecuado, a los fines epistémicos de la ciencia que, digamos, la determinación de dicho valor por una sola vía?" Algunas réplicas pueden parecer naturales: la coordinación de ambas mediciones muestra que hay algo en el mundo que se corresponde con las relaciones teóricamente postuladas. O bien: el hecho de que un mismo valor sea arrojado por dos o más vías diferentes no puede ser solo una coincidencia. Pero ninguna de estas posibilidades está disponible para el EE, ya que ellas hacen uso de la intuición del argumento del no-milagro, que indica que la mejor explicación del éxito predictivo de las teorías es su verdad aproximada. La pura metodología atada a un puñado de razones pragmáticas no es capaz de llenar la brecha que existe entre el modelo de datos y el fenómeno, más precisamente, no alcanza a justificar cómo se articula la relación de isomorfismo entre dos estructuras matemáticas y la representación de la realidad observable. El objetivo de la ciencia es epistémico, y la metodolo-

Principia 21(2): 473-482 (2017). 
gía es estéril si no se complementa con consideraciones extra-metodológicas que den cuenta del modo como ciertas restricciones normativas derivan en un conocimiento más ajustado de los fenómenos.

\section{Acerca de la problemática eficacia del CEG}

La razón por la que la pura metodología no es suficiente para tender un puente entre modelo de datos y fenómeno se vincula estrechamente con aquella detrás del fracaso de la primera respuesta a nuestra pregunta acerca de la justificación del CEG. Un criterio normativo para la práctica científica podría sustentarse en meras decisiones metodológicas referentes a la práctica científica si nos situáramos en un marco estrictamente convencionalista. Si el juego de la ciencia fuese, usando una expresión de Lakatos (1978, p.122), un juego como cualquier otro, la interacción entre su práctica y sus reglas (la metodología) no tendría por qué estar condicionada por presupuestos metafísicos, o por la satisfacción de metas epistémicas. Pero el escenario es bien diferente en el caso del EE. Su afirmación de un realismo del sentido común — que se ha mantenido incólume desde las primeras formulaciones del Empirismo Constructivo - y la elección de la adecuación empírica como objetivo de la ciencia determinan que la práctica científica está orientada hacia un fin epistémico concreto (i.e., un conocimiento creciente y acumulativo acerca de la estructura de los fenómenos), de este modo, la elección de una metodología es injustificada a menos que se muestre su eficacia con respecto a dicho fin. Ahora bien, si ninguna estructura objetiva impone restricciones a nuestras representaciones de los fenómenos, algo más debe hacerlo. El candidato de van Fraassen es el CEG, pero el problema es que las restricciones que éste impone no pueden vincularse con la satisfacción del objetivo epistémico de ganar un conocimiento (estructural) objetivo acerca de los fenómenos.

Creemos relevante notar que en su rechazo del Realismo Estructural, van Fraassen llega aún más lejos que en su eterna batalla contra realismo científico y lo que llamó "metafísica inflacionaria" (van Fraassen 1980, p.73). La tesis realista acerca de la existencia de las entidades inobservables postuladas por nuestras mejores teorías no es rechazada en favor de su opuesta, el instrumentalismo, sino reemplazada por una actitud agnóstica. Su rechazo de cualquier forma de realismo respecto de las estructuras, como hemos visto, es categórico: involucra la afirmación de que el mundo carece de toda estructuración objetiva.

No discutiremos aquí sus razones para sostener esta tesis. Nos limitaremos a sugerir la importancia de ofrecer un fundamento de la distinción entre representaciones adecuadas e inadecuadas. De ese modo, si admitimos que los fenómenos investigados por las teorías son un subconjunto de los objetos y procesos observables, entonces ese grano de realismo que van Fraassen está dispuesto a aceptar puede ser el hilo conductor que garantice el interjuego entre teorías, modelo de datos y fenómeno.

Principia 21(2): 473-482 (2017). 
Podríamos ser agnósticos respecto de cuál sea la estructura real de los fenómenos sin necesidad de convertirnos en ateos. En otras palabras, el agnosticismo que van Fraassen abraza hacia arriba - con respecto a la existencia de entidades y procesos inobservables - bien puede ser proyectado hacia abajo, no respecto de los fenómenos observables cuya existencia no admite dudas, sino con relación a algún patrón de comportamiento que no depende de nuestra mente, lenguaje o siquiera de nuestra existencia.

\section{Conclusiones}

Hemos procurado recorrer las nociones, distinciones y compromisos que fundan el EE a fin de dar cuenta de los profundos desafíos que enfrenta. Siguiendo a numerosos críticos, dimos cuenta de la objeción de pérdida de la realidad y de la propuesta vanfraassiana al respecto, a saber, el argumento de la indexicalidad. Sin embargo, en tanto estos tópicos han recibido suficiente atención en la literatura contemporánea, preferimos centrarnos en dos aspectos problemáticos adicionales: el problema del conocimiento objetivo y el problema de las restricciones a la representación. Como hemos visto, la necesidad de fundamentar la objetividad del conocimiento estructural de los fenómenos y la pretensión de salvaguardar una distinción entre representaciones adecuadas e inadecuadas son las principales motivaciones que impulsaron a van Fraassen a proponer el CEG.

Sin embargo, hemos mostrado que, pese a que el CEG aparece prima facie como un recurso legítimo para dar solución a dichas preocupaciones, su empleo en el contexto del EE no está suficientemente fundamentado ni resulta eficaz. En tanto conjunto de normas metodológicas, la apelación al CEG como garantía de la objetividad del conocimiento no puede ser justificada como un aspecto de la práctica científica ni como un medio orientado hacia la adecuación empírica de las teorías. En ambos casos, la legitimidad del CEG queda supeditada a la consecución de un objetivo epistémico concreto (i.e., un conocimiento estructural continuo y acumulativo sobre los fenómenos). Pero los compromisos básicos del EE hacen imposible brindar una visión plausible acerca de por qué el CEG es el criterio más adecuado (o siquiera un criterio adecuado) a tal fin.

Esta dificultad, con todo, no es nueva ni se limita a la formulación y uso del CEG. Las reglas metodológicas, por sí mismas, no constituyen una ruta directa hacia la justificación epistémica. A la hora de, por ejemplo, evaluar teorías científicas mediante procesos de contrastación se requiere de algún principio extra metodológico que haga converger el éxito empírico con el aumento del conocimiento. Lakatos entendió este punto antes y mejor que nadie. La metodología es ciertamente parte de la solución al problema de la objetividad del conocimiento, pero no puede ser toda la solución.

Principia 21(2): 473-482 (2017). 
Si bien nuestro objetivo primordial es crítico, creemos que una concesión mínima al realismo - que de todos modos se asume respecto de la existencia de los macro objetos cotidianos - podría orientar a van Fraassen hacia un camino fructífero en el tratamiento de estas cuestiones. Sugerimos que el agnosticismo, que históricamente ha sostenido respecto de las entidades inobservables, bien podría ser extendido hacia la existencia de ciertos patrones relacionales en los fenómenos.

\section{Referencias}

Borge, B.; Lucero, S. 2018. Ventajas y tensiones en la perspectiva del Estructuralismo Empirista. Revista de Filosofía 43(2). En prensa.

Lakatos, I. 1978. The methodology of scientific research programmes: Philosophical Papers. Vol.

I. J. Worrall; G. Currie (eds.) Cambridge: Cambridge University Press.

van Fraassen, B. 1980. The Scientific Image. Oxford: Clarendon Press.

\section{7.}

.2006. Representation: The problem for structuralism. Philosophy of Science 73(5): 536547.

Press.

- 2014. The Criterion of Empirical Grounding in the Sciences. In: W. González (ed.)

Bas van Fraassen's Approach to Representation and Models in Science, pp.79-100. Springer Netherlands.

Weyl, H. 1927[1963]. Philosophy of mathematics and natural science. New York: Atheneum.

SUSANA LUCERO

Universidad de Buenos Aires

Universidad Nacional de Luján

susanalucero8@fibertel.com.ar

BRUNO BORGE

Universidad de Buenos Aires

Consejo Nacional de Investigaciones Científicas y Tecnológicas brunojborge@gmail.com

\section{Notas}

${ }^{1}$ Van Fraassen explica la cuestión de cómo la teoría y las mediciones se hallan íntimamente relacionadas a través de un proceso que se ha ido constituyendo históricamente en conexión con varias otras teorías, hasta que finalmente la relación queda estabilizada. Cuando atendemos al proceso histórico, tenemos una mirada "desde adentro" (from within). Pero una vez que la relación teoría-mediciones se ha estabilizado, es la teoría la que determina la interrelación entre los parámetros, los instrumentos y los procedimientos para medir un fenómeno observable. Esta última es la mirada "desde arriba", desde la teoría (from above). Van Fraassen recomienda reunir las dos miradas en una visión sinóptica. (Van Fraassen 2008, pp.138-139).

Principia 21(2): 473-482 (2017). 